\title{
La interdisciplinariedad en el proceso de enseñanza-aprendizaje en postgrado. Una experiencia
}

\author{
Marga Vives ${ }^{a}$, Lydia Sánchez ${ }^{\text {b }}$ \\ ${ }^{\mathrm{a}}$ Universitat de les Illes Balears, Illes Balears, marga.vives@uib.es , ${ }^{\mathrm{b}}$ Universitat de les Illes Balears, \\ Illes Balears, lydia.sanchez@uib.es
}

\section{Resumen}

El aprendizaje en postgrado supone un reto didáctico de especialización universitaria que debe ser abordado; puesto que en un mismo máster podemos encontrar perfiles de alumnos de diferente procedencia de estudios y con experiencias laborales también variadas. Esta diversidad se amplia al participar en un máster con un doble itinerario: profesionalizador $e$ investigador.

Al mismo tiempo, debemos tener presente que dicho aprendizaje es de difícil fragmentación en asignaturas, puesto que todo un postgrado debe dotar de competencias, habilidades y conocimientos propuestos en su plan de estudios; claramente, el reto está en el equilibrio entre la transversalidad del ámbito de estudio y la especialización desde cada asignatura y que ésta sea percibida de forma correcta por todo el alumnado de postgrado.

Teniendo en cuenta estas premisas, se presenta una experiencia basada en esta interdisciplinariedad en el máster de intervención socioeducativa en menores y familia (MISO) de la Universitat de les Illes Balears, en concreto, a través de la asignatura "Mediación en contextos sociales y educativos"se vertebra un trabajo intra e interdependiente; por un lado, los trabajos dentro de la asignatura permiten un grado de especialización a los alumnos en determinados aspectos clave de la asignatura que desemboca, finalmente, en un trabajo grupal y que contempla el itinerario profesionalizador $e$ investigador. Por otro lado, se complementa con una vinculación directa con un taller de mediación realizado por un profesor universitario especialista en mediación y con un alto grado de experiencia laboral en este ámbito y con un vínculo directo con la asignatura "Técnicas de intervención educativas y conductuales en menores y familia” que se cursa en paralelo.

Palabras clave: Innovación educativa, interdisciplinariedad, universidad 


\section{Introducción}

Las instituciones educativas deben fomentar el incremento de actividades metodológicas, de estrategias didácticas diversas y de diseños curriculares, que sean dinámicas y adaptadas a las necesidades actuales y a los diferentes perfiles estudiantiles. Por consiguiente, se debe de apostar por métodos educativos que fomenten la interdisciplinariedad, porque es a partir de ésta que se podrán conseguir los anteriores objetivos metodológicos (Llano et al., 2016). La interdisciplinariedad podría ser definida como una puesta en común de saberes, una forma de conocimiento que se producen en la intersección de los saberes (Escobar \& Ramos, 2007). Además, hará referencia al método, al modelo y a la aplicación de la técnica. Por último, apuesta por procesos de aprendizaje desarrollados por diferentes docentes o profesionales, que, de manera conjunta, puedan aportar enfoques diversos (Bolarín, Moreno \& Porto, 2013; Vargas, Payrol, Hernández, \& Zambrana, 2018).

Las relaciones interdisciplinarias forman el método que posibilita mejorar, e incluso, optimizar, el proceso de enseñanza a los profesionales. El ámbito laboral, y las exigencias que tiene asociadas, implicará que los profesionales sean capaces de analizar la información que reciben de manera integral (Llano et al., 2016). Y que, además, deban responder de manera eficaz y resolutiva ante las diferentes demandas que emergerán. Por consiguiente, deben de estar provistos de un bagaje relevante de recursos y de conocimientos. El interdisciplinariedad permitirá construir esa perspectiva integral necesaria y un abanico de recursos obtenidos a partir de la aportación de diversas materias (Llano et al., 2016).

Como explica Souza Da Silva (2008), el concepto de interdisciplinariedad rompe con el modelo hegemónico en la producción científica, que destacaba la verdad como un objeto nuclear y que sólo abordaba los conocimientos que eran valorados como un producto acabado. La lucha por intentar acabar con esa rigidez en los modelos científicos, ha exigido la incorporación de nuevas perspectivas. La interdisciplinariedad permite reconocer nuevas fórmulas de aprendizaje, al considerar diferentes contextos académicos.

No obstante, también implica una cierta responsabilidad y retos. Su correcto uso y desarrollo implica que se conozcan las fronteras entre las diferentes disciplinas que deben de ser utilizadas (Souza Da Silva, 2008). Así como, que se aclaren y diseminan que aportaciones se pueden realizar si se combinan ambas disciplinas. Para conseguirlo, primero, se debe establecer donde convergen las disciplinas.

En el ámbito universitario, y específicamente, cuando se trata de educación de posgrado, es frecuente identificar heterogeneidad entre los perfiles de los estudiantes. El reto se inicia al intentar aportar conocimientos útiles y eficaces a los diferentes perfiles que se identifican. Cada tipología de estudiante ha adquirido conocimientos durante su formación universitaria 
y ha consolidado un aprendizaje asociado a su especialización. Además, la diversidad se podrá ver incrementa debido a que muchos alumnos de posgrado ya poseen experiencia en el mundo laboral. Por lo tanto, uno de los objetivos de las formaciones de posgrado es proporcionar equilibrio entre la transversalidad del ámbito de estudio y la especialización desde cada asignatura.

Al mismo tiempo, experiencias interdisciplinares permiten desarrollar competencia genéricas (potenciando el aprendizaje significativo) y comptencias específicas de cada una de las asignaturas que comparten este espacio interdisciplinar (Sandín, Lazo, Giménez \& Rodríguez, 2015).

Por lo tanto, los estudios de posgrado se convierten en un escenario por excelencia para fomentar el desarrollo integral del alumno. La interdisciplinariedad desde el ámbito universitario permite dotar de saber globales, fomentar la búsqueda de soluciones aplicables a otras disciplinas y encontrar un diálogo tanto para disciplinas cercanas o como más alejadas (Escobar \& Ramos, 2007; Vargas et al., 2018). Sin embargo, a pesar de su elevada recomendación, no es habitual identificar en los currículos vigentes la interdisciplinariedad (Escobar \& Ramos, 2007).

\section{Interdisciplinariedad en máster. Un ejemplo}

\subsection{Las asignaturas}

La asignatura "Técnicas de intervención educativas y conductuales en menores y familia" se establece como una asignatura obligatoria del máster MISO, impartida durante el segundo trimestre. Su finalidad principal consiste proporcionar y dotar de conocimientos, actitudes y estrategias orientadas a la intervención en menores y en familia. En concreto, se forma al alumnado en el uso y la aplicación de técnicas de intervención. Teniendo en cuenta que los alumnos son potenciales profesionales de atención directa a la familia (padres e hijos), es relevante que tengan herramientas suficientes para saber cómo actuar ante las posibles situaciones que puedan identificar. En concreto, las estrategias se dirigen a ser aplicadas en contextos sociales, educativos y familiares, y, por consiguiente, son de tipología educativa, cognitiva, conductual o emocional.

El diseño de la asignatura se establece en dos categorías principales:

a) Contextualización de los problemas de comportamiento y de las conductas no saludables. Este primer apartado, incorpora e identifica cuáles son los posibles factores de vulnerabilidad y de protección ante posibles trastornos, conductas problemas o conductas no saludables. Además, a partir de diferentes teorías y/o modelos teóricos explica los 
factores de predisposición y los factores de mantenimiento de los trastornos psicológicos. Así como establece posibles mecanismos inadecuados que emergentes de estructuras familiares disfuncionales o de pautas de crianza inadecuadas. Por consiguiente, en el primer apartado se establecen las bases teóricas y los conocimientos para realizar las evaluaciones de las situaciones en las que los profesionales deberán intervenir.

\section{b) Técnicas de intervención}

En este segundo apartado, se enseñan técnicas básicas de intervención para la modificación de patrones comportamentales inadecuados o de conductas problemáticas. Indudablemente, se enseñan técnicas de modificación de conducta; esenciales para la actuación directa con menores. En concreto, se identificarán aquellas dirigidas tanto al incremento, como a la disminución de conductas (extinción, reforzamiento, atención diferencial, coste de respuesta, tiempo fuera, modelado, moldeamiento, sobrecorrección, etc.). También se apuesta por estrategias de gestión emocional, donde se priorizará aquellas dirigidas a la relajación y el autocontrol. Además, se incorporan otras estrategias educativas para mejorar el clima en las escuelas y en el aula; un porcentaje relevante del alumnado son docentes o equipo orientativo. Por último, se realiza una aproximación a las técnicas cognitivas, como la reestructuración cognitiva (no obstante, el tiempo de la asignatura no permite profundizar en éstas últimas).

La asignatura "Mediación en Contextos Sociales y Educativos" se imparte también en el mismo master, de carácter obligatorio y en el mismo semestre que la asignatura con la que comparte experiencia educativa y comparten el mismo bloque temático "Intervención con menores y familia". Cuenta con 4 créditos ECTs y se trabaja, básicamente, en tres bloques temáticos:

En el primer bloque, se trabajan conceptos básicos sobre el conflicto: conceptualización, etiología y epistemiología de la convivencia. Habilidades para la gestión del conflicto (habilidades básicas para la gestión pacífica del conflicto, comunicación, empatía, escucha activa y asertividad). Se finaliza el bloque analizando tres modelos principiales de análisis del conflicto.

El segundo bloque se centra en la mediación; para ello, se inicia el bloque trabajando sobre estrategías de gestión del conflicto, como arbitraje, conciliación, negociación y mediación. Entrando en la mediación, se analizan las principales fases-objetivos, sus características básicas, modelos de mediación, hablidades y estrategías de los mediadores y se analiza código ético del mediador/a.

Finalmente, el tercer bloque aborda las características específicas de la mediación escolar, de la mediación familiar y de la mediación comunitaria. 


\subsection{Metodología}

Al ser un máster que dispone de un perfil profesionalizador e investigador, se plantean diferentes asignaturas para dar cabida a ambos perfiles. Al mismo tiempo, se pretende que todas las actividades estén relacionadas entre sí, de cara a que se entienda desde el primer momento la interconectividad.

En una primera fase, se trabaja en 3 tareas específicas:

1. Actividades en sesiones presenciales: se incorporan actividades prácticas en cada una de las sesiones presenciales que complementan las explicaciones y lecturas complementarias. La participación activa del alumnado en ella se evalua; en las últimas sesiones, por ejemplo, se realizan role-playings relacionados con cada ámbito de mediación y, si es posible, similares a los casos que, por grupos, los alumnos analizarán como trabajo grupal final.

2. Análisis y revisión de lecturas específicas: Se crearán grupos de 3 alumnos para la elaboración del trabajo final grupal; cada uno de los componentes realizará la lectura y análisis de un tema específico trabajado también en las sesiones presenciales: análisis del conflicto, fases/objetivos de la mediación y estrategias en mediación. La organización será la misma para todos los grupos de alumnos. La siguiente fase de la tarea convierte a cada alumno en revisor del trabajo previo de sus compañeros; así, si un alumno del grupo 1 ha analizado las fases, se convierte (de forma anónima) en revisor del trabajo realizado sobre las fases de su compañero del grupo X. La profesora se encarga de la distribución anónima de las revisiones a realizar y de devolver, también de forma anónima a cada alumno la revisión de su compañero para poder realizar la entrega final, resaltando los cambios realizados a partir de la revisión y comentarios adicionales a éstos. De esta manera, podemos trabajar ya con los alumnos la entrega de artículos y sus revisiones en el perfil académico.

3. Modelos en mediación: Finalmente, se analizan los modelos de mediación (tradicional, transformativo y circular-narrativo) siguiendo la misma dinámica de la actividad anterior; cada miembro del grupo realiza una lectura específica de cada modelo. En la sesión presencial, todos los alumnos se organizan según la lectura realizada (intergrupal), se comparan todos los modelos a través de una tabla comparativa y, al finalizar la sesión, cada grupo de trabajo grupal se reúne para evaluar qué tipo de modelo en mediación se adecua más a su caso práctico. 
A partir de estas actividades, que asientan las bases y competencias, se plantean tres actividades interdisciplinares:

1. Taller de mediación: realizado por un profesor invitado, se realiza un taller de mediación familiar que permite asentar los conocimientos y competencias trabajadas en las asignaturas.

2. Estrategías grupales: a partir de la escala de provención, se organizan grupos de 2-3 alumnos los cuales deben organizar una actividad relacionada con el tema correspondiente. En esta ocasión, la sesión se realiza en tiempo de la asignatura "Técnicas de intervención educativas y conductuales en menores y familia”, en su segunda hora y coincidiendo en día con la asignatura "Mediación en Contextos Sociales y Educativos", la cual continua en tercera y cuarta hora al terminar esta actividad de una hora de duración. Las actividades de la escala de provención que preparan los alumnos (organizados una semana antes para su elaboración) son: presentación, conocimiento, estima, confianza, comunicación y cooperación. La escala termina con la resolución de conflictos, pero no se realiza puesto que se trabaja de forma más específica en la asignatura de mediación.

3. Trabajo grupal: la tarea grupal encomendada engloba los principales conocimientos y competencias trabajados. A partir de un caso mediable, donde el grupo puede seleccionar el ámbito que desee (escolar, familiar o comunitario), deben realizar un análisis de conflicto, definir las fases/objetivos de la mediación y las principales estrategías que se ponen en marcha en su caso (correspondiente a la actividad 2 descrita anteriormente Análisis y revisión de lecturas específicas); seleccionar y describir el modelo de mediación sobre el que se basarán (actividad 3, Modelos en mediación) y analizar y describir el tipo de mediación (a partir de las sesiones presenciales, actividad 1 y lecturas específicas). El trabajo se completa con la descripción del caso, la evaluación de la mediación, conclusiones y autoevaluación y evaluación del grupo.

De cara a valorar los resultados, se realizó un cuestionario a los alumnos que incluía su perfil sociodemográfico y vinculación con el mundo laboral con familias y menores y un segundo bloque con la valoración de esta experiencia didáctica. Finalmente, se comprobaron también los resultados de aprendizaje a través de una prueba pre-post coincidende con el inicio y el final de la asignatura de "Mediación en contextos sociales y educativos". 


\subsection{Resultados}

El grupo de alumnos de este curso académico (18-19) es altamente femenino (80\%), donde un $70 \%$ ha trabajado o trabaja como profesional en atención en menores y familias (entre un año -30\%- o más de 5 años -30\%-).

La mayoría de alumnos recomiendan la combinación de asignaturas para el aprendizaje (75\%), donde básicamente se valora la autoreflexión y participación en clase $(37,5 \%)$ y la explicación docente de calidad (25\%). La opción preferida por los alumnos es la realización grupal de un caso práctico $(50 \%)$ y de actividades prácticas en clase $(25 \%)$.

Este cuestionario fue complementado con una revisión de la evaluación inicial en la asignatura de "Mediación en contextos sociales y educativos", donde se ha podido observar la afianzación de contenidos así como la adquisición de aquellos no consolidados.

\section{Conclusiones}

El aprendizaje interdisciplinar permite trabajar, desde diferentes perspectivas, la adquisición de unas competencias profesionales e investigadoras desde diferentes perspectivas y metodologías de aprendizaje; en este sentido, debe contemplar un doble objetivo, por un lado, asegurar la adquisición de competencias específicas de cada asignatura y, al mismo tiempo, potenciar la consolidación de competencias generales trabajadas desde diferentes asignaturas (Sandín et al, 2015). Todo ello, teniendo en cuenta, en el caso de nuestro ejemplo, los perfiles profesionales e investigadores que permite el máster así como la adaptabilidad al perfil académico y profesional de cada una de las promociones del máster.

Desde la experiencia explicada, hemos querido aportar unas bases para la reflexión sobre algunas propuestas para poder adquirir contenidos y competencias específicas en un máster de intervención socioeducativa en menores y familia con su aplicabilidad en el ámbito profesional (Escobar \& Ramos, 2007), centrada en un aprendizaje global (Vargas et al., 2018).

\section{Referencias}

Bolarín, M. J., Moreno, M. Á., \& Porto, M. (2013). Coordinación docente e interdisciplinariedad: análisis de su contribución a la adquisición de competencias docentes y discentes. REDU. Revista de Docencia Universitaria, 11(2), 443-462. 
Escobar, A. E. V., \& Ramos, I. O. (2007). VI. Enseñar-aprender para el desarrollo: la interdisciplinariedad como una alternativa de solución. Pedagogía Universitaria, 12(2). Disponible en $\quad$ http://pepsic.bvsalud.org/scielo.php?script=sci_arttext\&pid=S1870$\underline{350 \times 2008000300014}$

Llano, L., Gutiérrez, M., Stable, A., Núñez, M., Masó, R., \& Rojas, B. (2016). La interdisciplinariedad: una necesidad contemporánea para favorecer el proceso de enseñanza aprendizaje. Medisur, 14(3), 320-327.

Sandín, M.; Lazo, X.; Giménez, M. \& Rodríguez, M. (2016) Trabajando la integración interdisciplinar: Evaluación de una actividad puente entre dos asignaturas del Grado en Ciencias Ambientales REDU. Revista de Docencia Universitaria, 14(1), 245-260.

Souza Da Silva Batista, S. H. (2008). Interdisciplinariedad, docencia universitaria y formación. Educación Médica Superior, 22(4), Disponible en: http://scielo.sld.cu/pdf/ems/v22n4/ems07408.pdf

Vargas, I. R., Payrol, J. A., Hernández, G. C. C., \& Zambrana, C. L. C. (2018). La interdisciplinariedad en la gestión pedagógica, una tarea de los profesores de la universidad actual. Boletín Redipe, 7(8), 89-97. 